\title{
Health sector reform
}

No one is too clear about the underlying goals of health sector reforms. The cynical may detect a neo-liberal free market agenda in the rhetoric that emanates from the World Bank's health report of 1993, ${ }^{1}$ but even the cynical need to deal with the issues that health reforms throw up. More recently the Bank itself has recognised the failures of the market approach in health care and argued for a greater involvement of the state in the health sector. ${ }^{2}$ What are the appropriate goals for public authorities and for the state in reforming the health sector, and is there any consensus on these goals? The health sector reform work programme underway at Liverpool School of Tropical Medicine has outlined some possible goals: health gain, by curative and preventive care; equity, by fair distribution of benefits provided by society and the state; social care, by looking after those who cannot be cured; insurance, by protecting individuals and households against health care costs; and the national economy, by ensuring a healthy workforce.

There is no consistently applied, universal package of measures that constitutes health sector reform. Rather, the precise agenda for reform will be defined by reviewing how well existing policies, institutions, structures, and systems deal with issues of efficiency, access, cost containment, and responsiveness to popular demand. ${ }^{3}$ The relative importance of these issues will vary between less developed countries, industrialised countries, and countries in transition from a command economy. In less developed countries, reform strategies need to address the issues of extending the coverage of basic services to under-served populations, improving poor service quality, and addressing the inequitable distribution of resources, in the context of very limited institutional capacity. In many of the world's richer countries cost containment has been the driving force behind reform. However, the need for systems to ration health care provision in line with national policy objectives is common to all countries.

Each country has its own agenda for health sector development, but three broad policy objectives usually feature. ${ }^{4}$

IMPROVING HEALTH STATUS

Health care should improve the health of the population. Although the potential of health services in this respect remains unclear, much of the current fashion for cost effectiveness analysis is directed by this goal of achieving the maximum improvement in population health status by the systematic allocation of the limited resources available for health care.

IMPROVING EQUITY AND REDUCING POVERTY

This goal shifts the emphasis to who benefits from public spending on health, arguing that such spending should differentially benefit the poor rather than simply those who suffer from conditions with cost effective interventions available. This approach therefore values health care as an instrument for poverty alleviation, in line with the shift now being seen in the policy of several donor agencies.

\section{IMPROVING INDIVIDUAL WELFARE}

Clearly health is something that people value (though not as highly as the readers of this journal might think). The behaviour of individuals in seeking and purchasing health services reflects that valuation. A government pursuing the goal of improving individual welfare might limit public action to those services for which there are substantial market failures, and leave other services to individual judgement and the private market.
Judged by these criteria, I would argue that one of the most successful reforms was the construction of a truly National Health Service in the UK. No one doubts the tremendous improvement in access to care and quality of service brought about by this reform, and few serious observers even now argue with the cost of such a reform, which remains among the lowest in the developed world (expressed as a percentage of GDP). We would do well to remember in the modern enthusiasm for overnight solutions to age old problems that the foundations of the NHS were laid nearly a century ago in the legislation preceding various welfare reforms.

It is convenient to divide reform actions into four main domains. The first domain is the actual package of services provided. Identifying a package of essential services has become a priority for the reform agendas of many developing countries, encouraged by the international donors' view that universal access to health care is unsustainable for the world as a whole. The second domain is finance, which drives the push to reduce the subsidy and redistribution of resources to the poor that is characteristic of public expenditure on health services. (Such a requirment of course is central to the Structural Adjustment Programme initiative that so many developing countries have been subjected to.) These concerns about financing of health services have implications at the organisational level, which is the third domain and has unexpected influences on both supply and demand. The fourth domain is that of user concerns and perspectives. The organisation of health care unfortunately responds very sluggishly to these concerns, and little in the focus of the current enthusiasm for health reforms leads me to be optimistic that this situation is changing.

One important feature of policies for the health sector is their implicit allocation of budgets based on the priorities they identify. These allocations will necessarily depend upon the relative weight given to these different domains of health policy goals. But they do not form the whole picture of the allocation rules. Resource allocation will also depend upon the constraints facing governments, particularly the market environment within which they operate. For example, the pricing policy of international pharmaceutical companies has a substantial influence on health policies. The cost effectiveness of antiretroviral treatments for HIV may be debated in developed countries while the treatments themselves are being evaluated in poorer countries where they will remain unaffordable unless the drug companies change their own policies and become true partners in the battle against AIDS.

It is also clear that the process of change needs to extend beyond the redefinition of policy objectives and discussions of the ideological orientation of the health care system. Without institutional or structural change it is likely that existing organisational structures and management systems will continue to fail to deal adequately with the problems listed above. Health sector reform will therefore be concerned with defining priorities, refining policies and reforming the institutions through which those policies are implemented. ${ }^{5}$

The process of reform and the difficulty of implementing policy and institutional change has been relatively neglected compared with the debate about the content of reform. This focus on content not only ignores the question of the feasibility of implementing change, but runs the risk that health sector reform becomes equated 
with one particular set of prescriptions - such as the introduction of managed-market mechanisms, user charges, reducing the size of the public sector, cost effective packages of services, and privatisation. As a result the need for creative solutions to deal with urgent and intractable problems can easily get lost in discussions about the rights and wrongs of particular strategies.

There is a need for rational debate and systematic analysis. In the first instance, this requirement must be addressed by descriptive information on reforms using a taxonomy that aids the analysis of the implementation and impact of reforms. Such a framework should allow a synthesis of the benefits and drawbacks of reforms that can assist each country's attempts at producing better health from the level of investment within that country.
Liverpool School of Tropical Medicine

The Health Sector Reform Work Programme is a five year programme of research and analysis that focuses on the impact of reforms on the quality, Department for International Development (formerly ODA).

1 World Bank. World Development Report 1993: Investing in health. New York: Oxford University Press, 1993.

2 World Bank. World Development Report 1997: The state in a changing world. New York: Oxford University Press, 1997.

3 Cassels A. A guide to sector-wide approaches for health development: concepts, issues and working arrangements. Geneva: World Health Organization, 1997.

4 Berman P, ed. Health sector reforms in developing countries: making health development sustainable. Boston: Harvard University Press, 1995.

5 Cassels A. Health sector reform: key issues in less developed countries. Fournal of Interntional Development 1995;3:329-47. 